\title{
A comparison of Monte Carlo and analytic first scatter dose spread arrays
}

\author{
J. E. McGary a) and A. L. Boyer ${ }^{\text {b) }}$ \\ Department of Radiation Physics, The University of Texas M. D. Anderson Cancer Center, Houston, \\ Texas 77030 \\ T. R. Mackie \\ University of Wisconsin, Madison, Madison, Wisconsin 53706
}

(Received 14 December 1998; accepted for publication 3 March 1999)

\begin{abstract}
We compare first scattered point dose spread arrays generated by Monte Carlo and an analytic method. The analytic method models energy deposition using Klein-Nishina cross sections for Compton scatter and approximations for electron transport. Assumptions in the analytic method are shown to be valid within a region of the point dose spread kernel in which meaningful comparisons can be made. Differences between the models are less than $10 \%$ for the forward scatter directions for radii greater than the electron range associated with the first scattered Compton photon. Differences in the backscatter region are discussed and indicate that the analytic model is useful for identifying large errors that might be present in numerically generated first scatter point dose spread arrays. The analytic method is simple and useful for validating first scatter kernels. (C) 1999 American Association of Physicists in Medicine. [S0094-2405(99)02305-6]
\end{abstract}

Key words: Monte Carlo simulation, convolution calculation, kernels

\section{INTRODUCTION}

Three-dimensional photon dose distributions can be calculated using convolution techniques. ${ }^{1-4}$ The technique spatially convolves the primary fluence with a kernel that describes the transport and energy deposition by secondary particles. The convolution kernel has been termed the point dose spread array due to the spread of dose throughout a volume surrounding the point at which the interaction of the primary photon occurs. Alternatively, the kernel has been called a "differential pencil beam'" to describe the dose distribution around an infinitesimally narrow beam.

Point dose spread arrays have been calculated with Monte Carlo (MC) methods to describe the energy deposition from electrons and scattered photons. In particular, Monte Carlo simulations were performed using the EGS4 Monte Carlo code to model different contributions according to the scatter order which originate at the interaction site from an incident monoenergetic photon. ${ }^{5}$ The separate contributions include the primary, first, second, and multiple scatter components. Energy transport from the primary interaction site to the surrounding dose deposition voxels is accounted for by using the following scoring categories: primary energy deposited by electrons and positrons set in motion by the incident monoenergetic photon interaction at the primary site; first scattered Compton photons originating at the primary site; and similarly for second and multiple scatter photons. Contributions from bremsstrahlung and annihilation photons were scored as a separate category resulting from all scattering orders. The complete dose spread array is the sum of the separate scatter components and can be referred to as the kernel of the convolution, even though the calculation may be performed by convolving the fluence with the separate kernel components and then summed together for the resulting dose distribution. ${ }^{6}$
Monte Carlo generated dose spread arrays have been used in a variety of applications but have not received extensive verification. The complete dose kernel has been indirectly tested by comparisons with experimental measurements taken from beam features such as depth dose data, tissue maximum ratios, and beam profiles. In a more direct approach, it has been suggested that the total energy deposition kernel could be obtained from deconvolving measured narrow beam dose distributions. ${ }^{7,8}$ Furthermore, a semiempirical method was developed to characterize a pencil beam dose kernel and then compared with Monte Carlo calculations. ${ }^{9}$ Information gained from these comparisons is useful for determining the total uncertainty within a dose calculation but provides little insight into the source of errors that might be present within the dose kernel. In contrast, validating the individual dose spread arrays of the kernel establishes the accuracy of each component and provides a guide for modeling improvement and increased accuracy. To validate these components, Monte Carlo results should be compared with other Monte Carlo simulations, experimental measurements, and other calculations that model the same physics. Monte Carlo results may be compared among experienced users to analyze differences due to algorithm or numerical variations to derive accuracy limits for using this type of method. Experimental results are an important method for comparison in that they provides independent verification of the dose spread arrays. Some of these experiments have been performed to test the primary dose spread array. O'Connor and Malone derived the primary point dose spread array from experimental measurements using cobalt-60 gamma rays and compared them with published MC data. ${ }^{10}$ They suggested methods for performing accurate measurements of higher energy photons and dose contributions from scattered photons by extending their technique. To complement these compari- 


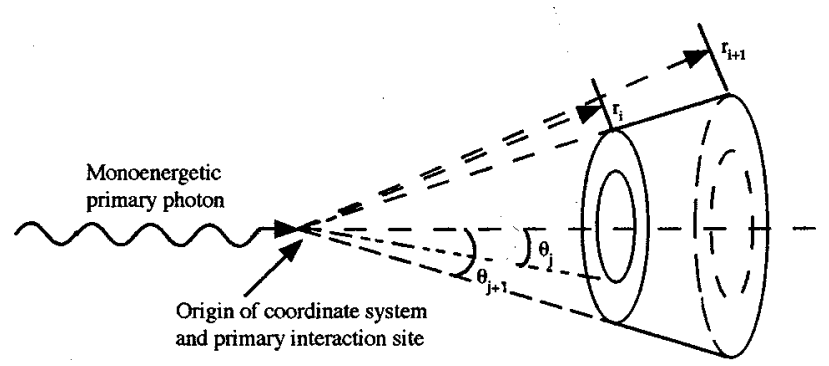

FIG. 1. Spherical geometry defined for calculation showing an energy deposition voxel bounded in radius and polar angle.

sons, various calculations have been made that model the primary and scatter contributions but they compared their results with the resulting dose distributions and not the individual dose spread arrays. ${ }^{11,12}$

In an effort to help validate the first Compton scatter point dose spread array, we developed two simple analytic models to directly compare with the Monte Carlo generated kernel component. The analytic models are designed to investigate the plausibility of the MC first scatter dose array and test the accuracy in the regions where the analytic model assumptions are shown to be valid. The analytic expression for energy deposition from the first scattered Compton photon uses the well-known Klein-Nishina scattering cross sections to calculate the photon scatter probability, and two extreme conditions for electron transport are considered. The results are compared with the first Compton scatter point dose spread arrays published by Mackie et al. ${ }^{5}$

\section{METHODS}

The geometry used in the Monte Carlo model and analytic calculation is based on a spherical $(r, \theta, \phi)$ coordinate system where the primary interaction site defines the origin, $\theta$ defines the polar angle away from the incident photon direction, and $\phi$ is the azimuth angle. The energy deposition kernels are determined for voxels defined by the intersection of 48 cones, separated by a polar angle of $3.75^{\circ}$, and 24 spherical shells as shown in Fig. 1. Rotational symmetry is assumed about the axis defined by the incident photon direction and the shell thickness varies with radius: the voxel length is $10 \mathrm{~cm}$ for radii greater than $10 \mathrm{~cm}$; for radii less than $10 \mathrm{~cm}$, the voxel length varies between 1 and $2 \mathrm{~cm}$; and for radii less than $1 \mathrm{~cm}$, the voxel length ranges from 0.05 to $0.1 \mathrm{~cm}$.

The monoenergetic incident photon fluence, $\Phi_{0}$, of energy $h \nu_{0}[\mathrm{MeV}]$ interacts within a thin slab centered about the origin with area $A_{0}$ and infinitesimal thickness $d z$. This will be called the interaction site since the slab dimensions are small compared with that of the voxels. The probability of scattering $d N_{s}$ photons into a solid angle $d \Omega$ per incident photon fluence per electron for Compton scattering is given by

$$
\begin{array}{r}
\frac{1}{\Phi_{0}}\left(\frac{d N_{s}\left(h \nu^{\prime}(\theta), \theta, \phi\right)}{d \Omega(\theta, \phi)}\right) d \Omega(\theta, \phi) \\
=\left(\frac{d \sigma\left(h \nu_{0}, \theta, \phi\right)}{d \Omega(\theta, \phi)}\right) d \Omega(\theta, \phi),
\end{array}
$$

where $(\theta, \phi)$ are the spherical angles describing the conical wedge that the photons are scattered into using the KleinNishina differential cross section for Compton scattering with energy of the scattered photons given by $h \nu^{\prime}(\theta)$ $[\mathrm{MeV}]$. The number of scattering centers at the interaction site is $\rho_{e}^{\prime} A_{0} d z$, with $\rho_{e}^{\prime}$ defined as the electron density (electron $/ \mathrm{cm}^{3}$ ) in the interaction voxel. For azimuthal symmetry, the total number of photons scattered into a differential solid angle from the interaction site is given by

$$
\begin{aligned}
& \left(\frac{d N_{s}\left(h \nu^{\prime}(\theta), \theta\right)}{d \Omega(\theta)}\right) d \Omega(\theta) \\
& \quad=\Phi_{0} \rho_{e}^{\prime} A_{0} d z \times\left(\frac{d \sigma\left(h \nu_{0}, \theta\right)}{d \Omega(\theta)}\right) d \Omega(\theta) .
\end{aligned}
$$

Along the radial direction, the number of photons within the annulus defined by $(r, \theta)$ and $(r, \theta+d \theta)$ is equal to the number of photons scattered into the differential solid angle at the origin attenuated through absorption and scattering along the path between the primary interaction site and annulus. This is described in terms of the linear attenuation coefficient $\mu\left(h \nu^{\prime}\right)\left(\mathrm{cm}^{-1}\right)$ and radial distance $r(\mathrm{~cm})$ :

$$
\begin{aligned}
& \left(\frac{d N_{s}\left(h \nu^{\prime}(\theta), r, \theta\right)}{d \Omega(\theta)}\right) d \Omega(\theta) \\
& =\Phi_{0} \rho_{e}^{\prime} A_{0} d z \times\left(\frac{d \sigma\left(h \nu_{0}, \theta\right)}{d \Omega(\theta)}\right) d \Omega(\theta) \times e^{-\mu\left(h v^{\prime}\right) r} .
\end{aligned}
$$

\section{A. Zero range approximation}

If we assume that the scattered photons are absorbed through interactions and deposit their energy at the interaction point, then the energy deposited in a small volume element is equal to the number of absorbed photons within the element times their energy:

$$
d E_{a}=h \nu^{\prime}(\theta) \times d N_{a}\left(h \nu^{\prime}, r, \theta\right),
$$

where the number of absorbed photons, using the energy absorption coefficient $\mu_{a}\left(h \nu^{\prime}\right)\left(\mathrm{cm}^{-1}\right)$ is expressed by

$$
\begin{aligned}
d N_{a}\left(h \nu^{\prime}(\theta), r, \theta\right)= & \left(\frac{d N_{s}\left(h \nu^{\prime}(\theta), r, \theta\right)}{d \Omega(\theta)}\right) d \Omega(\theta) \\
& \times \mu_{a}\left(h \nu^{\prime}\right) d r .
\end{aligned}
$$

The energy deposited in an infinitesimal volume element described by a small conical wedge is found by using Eqs. (3)-(5):

$$
\begin{aligned}
d E_{a}(r, \theta)= & h \nu^{\prime}(\theta) \Phi_{0} \rho_{e}^{\prime} A_{0} d z \times\left(\frac{d \sigma\left(h \nu_{0}, \theta\right)}{d \Omega(\theta)}\right) \\
& \times e^{-\mu\left(h \nu^{\prime}\right) r} \times \mu_{a}\left(h \nu^{\prime}\right) d \Omega(\theta) d r .
\end{aligned}
$$


If we normalize the deposited energy to the total energy of the incident primary photons that interact at the primary interaction site, then we get an equation describing the fractional amount of energy deposited in a volume element:

$$
\begin{aligned}
d \varepsilon_{a}(r, \theta)= & \frac{d E_{a}(r, \theta)}{\Phi_{0} A_{0} \times \mu\left(h \nu_{0}\right) d z \times h \nu_{0}} \\
= & \frac{h \nu^{\prime}(\theta)}{h \nu_{0}} \rho_{e}^{\prime} \times\left(\frac{d \sigma\left(h \nu_{0}, \theta\right)}{d \Omega(\theta)}\right) \times e^{-\mu\left(h \nu^{\prime}\right) r} \\
& \times \frac{\mu_{a}\left(h \nu^{\prime}\right)}{\mu\left(h \nu_{0}\right)} d \Omega(\theta) d r .
\end{aligned}
$$

This equation can be integrated to calculate the fractional energy deposited within a voxel bounded by $\left(r_{\mathrm{i}-1}, r_{\mathrm{i}}\right)$, and $\left(\theta_{j-1}, \theta_{j}\right)$, and the corresponding fractional dose associated with that voxel is then determined by dividing the fractional energy deposited by the mass of the voxel:

$$
\begin{array}{r}
D_{i, j}=\frac{\int_{\theta_{j-1}}^{\theta_{j}} \int_{r_{i-1}}^{r_{i}} d \varepsilon_{a}(r, \theta)}{M_{i, j}} \mathrm{cGy} / \mathrm{MeV} \text { photon, } \\
\text { where } M_{i, j}=\int_{\theta_{j-1}}^{\theta_{j}} \int_{r_{i-1}}^{r_{i}} \rho d \operatorname{vol}(r, \theta) .
\end{array}
$$

Equation (8) is solved numerically using analytic expressions for all quantities except for the linear attenuation and energy absorption coefficients where table look up is used..$^{13}$ For comparison with the Monte Carlo data, the calculation extends from $r=0-50 \mathrm{~cm}$ and $\theta=0-170^{\circ}$.

\section{B. Finite range approximation}

In comparison with the previous assumption that the energy is deposited at the scattered photon-electron interaction site, we now consider modeling an effect of electron transport on the energy deposition kernels. The zero-range model should be useful for conditions where lateral electronic equilibrium exists; however, near the primary interaction site this assumption is not valid. To estimate the energy deposited in this region, we derive a simple analytic formulation that includes energy deposition from electrons created by the first scattered photons where transport is restricted along the radial direction. The energy deposited in a volume element at $(r, \theta)$ can be expressed in terms of the number of the in-range electrons created upstream at $\left(r^{\prime}, \theta\right)$, the path length traversed by the electrons within the volume element, and the collision stopping power:

$$
d E(r, \theta)=-d N_{e}\left(r^{\prime}, \theta, h v^{\prime}(\theta)\right) \times S_{c}\left(E_{e}\left(r-r^{\prime}\right)\right) h(r) .
$$

This includes contributions from electrons, generated by scattered photons of energy $h v^{\prime}$, within the practical range of $r$ that defines the lower boundary of the volume element. The collisional stopping power $S_{c}$, depends on the average electron energy transferred from the scattered photon $E_{\mathrm{e}}\left(r-r^{\prime}\right)$, which is a function of distance $r-r^{\prime}$ traveled by the electron from the interaction point. The function $h(r)$ represents a window length of $R_{p}$ that extends from the elec- tron creation point $r^{\prime}$ to distance $r^{\prime}+R_{p}$. The window function $H(r)$, is generated by the combination of two Heaviside step functions and described by

$$
h(r)=\left\{H\left(r-r^{\prime}\right)-H\left(r-\left(r^{\prime}+R_{p}\right)\right\} .\right.
$$

$H\left(r-r^{\prime}\right)$ is zero for values of $r$ less than $r^{\prime}$ and equal to 1 for $r>r^{\prime}$. Integrating Eq. (9) over the radial voxel boundaries and the electrons created along the radial direction gives the total energy deposited within a volume element:

$$
\begin{aligned}
E(r, \theta)= & -\int_{r_{i-1}-R_{p}}^{r_{i}} d N_{e}\left(r^{\prime}, \theta, h v^{\prime}(\theta)\right) \\
& \times \int_{r_{i-1}}^{r_{i}} S_{c}\left(E_{e}\left(r^{\prime \prime}-r^{\prime}\right)\right) \cdot h\left(r^{\prime \prime}\right) d r^{\prime \prime} .
\end{aligned}
$$

The lower limit of the first integral restricts counting electrons beyond the practical range of reaching the deposition voxel; however, Eq. (11) could be integrated over all $r$ since the window function eliminates contributions from electrons at distances greater than the practical range from the boundary of the voxel. The electrons $d N_{\mathrm{e}}\left(r^{\prime}, \theta\right)$ are created from first scattered photon interactions at $r^{\prime}$, and as in Eq. (5), $d N_{\mathrm{e}}\left(r^{\prime}, \theta\right)$ is described in terms of the linear attenuation coefficient as

$$
\begin{aligned}
d N_{e}\left(r^{\prime}, \theta, h v^{\prime}(\theta)\right)= & \Phi_{0} A_{0} \rho_{e}^{\prime} d z \times\left(\frac{d \sigma\left(h \nu_{0}, \theta\right)}{d \Omega(\theta)}\right) d \Omega(\theta) \\
& \times \mu\left(h \nu^{\prime}\right) \times e^{-\mu\left(h \nu^{\prime}\right) r^{\prime}} d r^{\prime} .
\end{aligned}
$$

Using the same definitions discussed in the previous section, the expression for the fractional dose associated with a given voxel is

$$
\begin{aligned}
D_{i, j}= & \frac{-\rho_{e}^{\prime}}{\mu\left(h \nu_{0}\right) h \nu_{0} M_{i, j}} \int_{\theta_{j 1}}^{\theta_{j}}\left(\frac{d \sigma\left(h \nu_{0}, \theta\right)}{d \Omega(\theta)}\right) \mu\left(h \nu^{\prime}\right) d \Omega(\theta) \\
& \times \int_{r_{i-1}-R_{p}}^{r_{i}} e^{-\mu\left(h \nu^{\prime}\right) r^{\prime}} d r^{\prime} \int_{r_{i-1}}^{r_{i}} S_{c}\left(E_{e}\left(r^{\prime \prime}-r^{\prime}\right)\right) \\
& \times h\left(r^{\prime \prime}\right) d r^{\prime \prime} .
\end{aligned}
$$

To solve Eq. (13), we assume that electrons lose energy linearly with distance. A relation of the electron energy as a function of distance from the creation point $r^{\prime \prime}-r^{\prime}$, can be expressed as

$$
E_{e}\left(r^{\prime \prime}-r^{\prime}\right)=\frac{E_{e}\left(r^{\prime}\right)}{R_{p}} \times\left\{\left(R_{p}+r^{\prime}\right)-r^{\prime \prime}\right\},
$$

where $R_{p}$ is a function of $E_{\mathrm{e}}\left(r^{\prime}\right)$. Accounting for bremsstrahlung, the collisional stopping power in Eq. (13) is determined from the total stopping power which is found using Eq. (14) and written as

$$
S_{t}\left(E_{e}\left(r^{\prime \prime}-r^{\prime}\right)\right)=\frac{\partial E_{e}}{\partial r^{\prime \prime}}=-\frac{E_{e}\left(r^{\prime}\right)}{R_{p}\left(E_{e}\left(r^{\prime}\right)\right)},
$$

where $E_{\mathrm{e}}\left(r^{\prime}\right)$ is equal to the mean energy transferred per photon interaction $E_{\mathrm{tr}}$. Pair production electrons are included in these calculations with a mean energy transferred, 


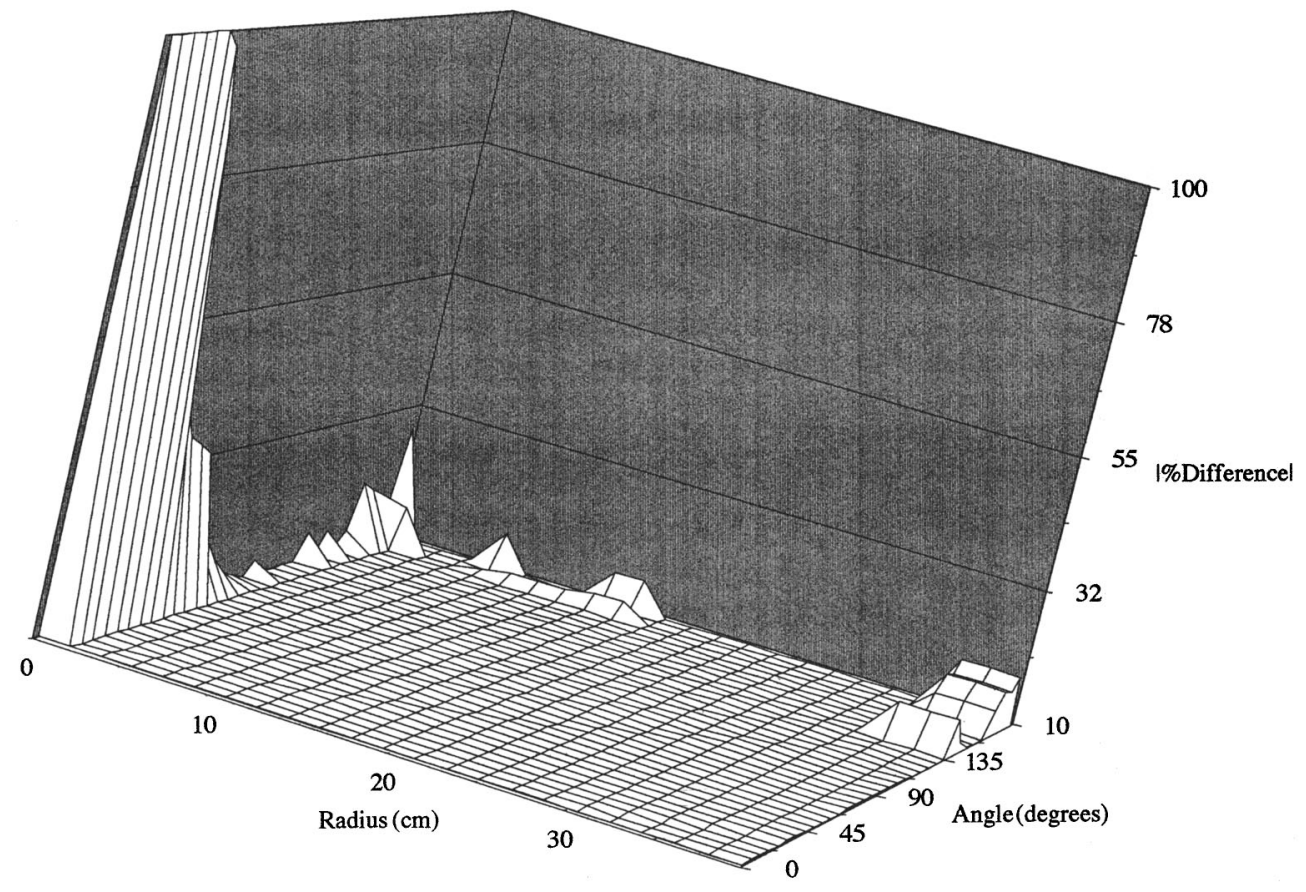

FIG. 2. Comparison of the zero-range analytic model and Monte Carlo results for the $1.25 \mathrm{MeV}$ first scatter dose spread array. The absolute differences between models are plotted as a function of radius and scatter angle.

per electron, that is equal to $\left(h \nu^{\prime}-1.02\right) / 2$. Contribution from Compton and pair production electrons are calculated separately according to their range and energy. Number densities for each electron species is determined from the interaction cross sections and total linear attenuation coefficients. Cross section values and linear attenuation coefficients are calculated from interpolation using table look up. ${ }^{13}$

\section{RESULTS}

We chose three incident monoenergetic photon energies for comparison between the analytic and Monte Carlo first Compton scatter point dose spread array: 1.25, 5.0, and 18 $\mathrm{MeV}$. The Monte Carlo data used for these comparisons were taken from calculation results generated by Mackie et al..$^{5}$ and subtracted from the analytic model calculations to determine absolute percent differences. The absolute percent differences are represented by surface plots using the coordinate system described in Fig. 1. The origin of the coordinate system is defined as the incident monoenergetic photon interaction site and the polar angle is the Compton photon scattering angle. The radial axis extends to a $40 \mathrm{~cm}$ radius and the scatter angle ranges from $0^{\circ}$ to $170^{\circ}$. All plots are labeled between $10 \%$ and $100 \%$ to emphasize differences greater than $10 \%$. The surface plots are best for demonstrating differences over several voxels, but not individual voxels due to interpolation. For large fluctuations that occur between voxles, the values shown in the graphs will be smaller than the actual differences. Typically, these fluctuations and inaccuracies in the plots are found in the backscatter region for angles larger than $150^{\circ}$.

Comparisons between the Monte Carlo and analytic first scatter dose spread arrays for $1.25 \mathrm{MeV}$ incident photons are shown in Figs. 2 and 3. Figure 2 shows the differences between the zero range analytic model and the MC first scatter kernel. The largest differences occur near the origin for radii less than the electron range associated with a $1.25 \mathrm{MeV}$ photon. For angles less than $5^{\circ}$, the percent difference between the zero range model and MC dose spread array is less than 1000. In contrast, Fig. 3 demonstrates that the finite-range model, for the $1.25 \mathrm{MeV}$ incident photon, approximates the MC kernel data in the region near the origin. The finite-range model accounts for electron transport radially away from the origin, whereas the zero-range model assumes energy deposition at the scattered photon-electron interaction point, and therefore, overestimates the energy deposition in voxels near the origin. Away from the origin, both analytic models agree with the Monte Carlo scatter kernel data to within $10 \%$ for most of the volume considered in these calculations. Other notable differences between the analytic models and the Monte Carlo data occur in the backscatter region. The Monte Carlo data decrease as a function of polar angle, oscillating and exhibiting large fluctuations in the backscatter region, while the analytic models decreases smoothly with radius and scatter angle. There are several voxels, located near the origin for angles larger than $160^{\circ}$, that exhibit differences as large as $70 \%$ in the data file but are only shown in the plot to be $\sim 35 \%$ as a result of interpolating from a nonlinear calculation grid.

Comparisons between model calculations for $5 \mathrm{MeV}$ incident photons are presented in Figs. 4 and 5. A comparison between the zero-range model and the Monte Carlo first scatter dose spread array, Fig. 4, demonstrates similar behavior as seen in the previous comparison for the $1.25 \mathrm{MeV}$ first scatter kernel data. The $5 \mathrm{MeV}$ first scatter kernel calculated 


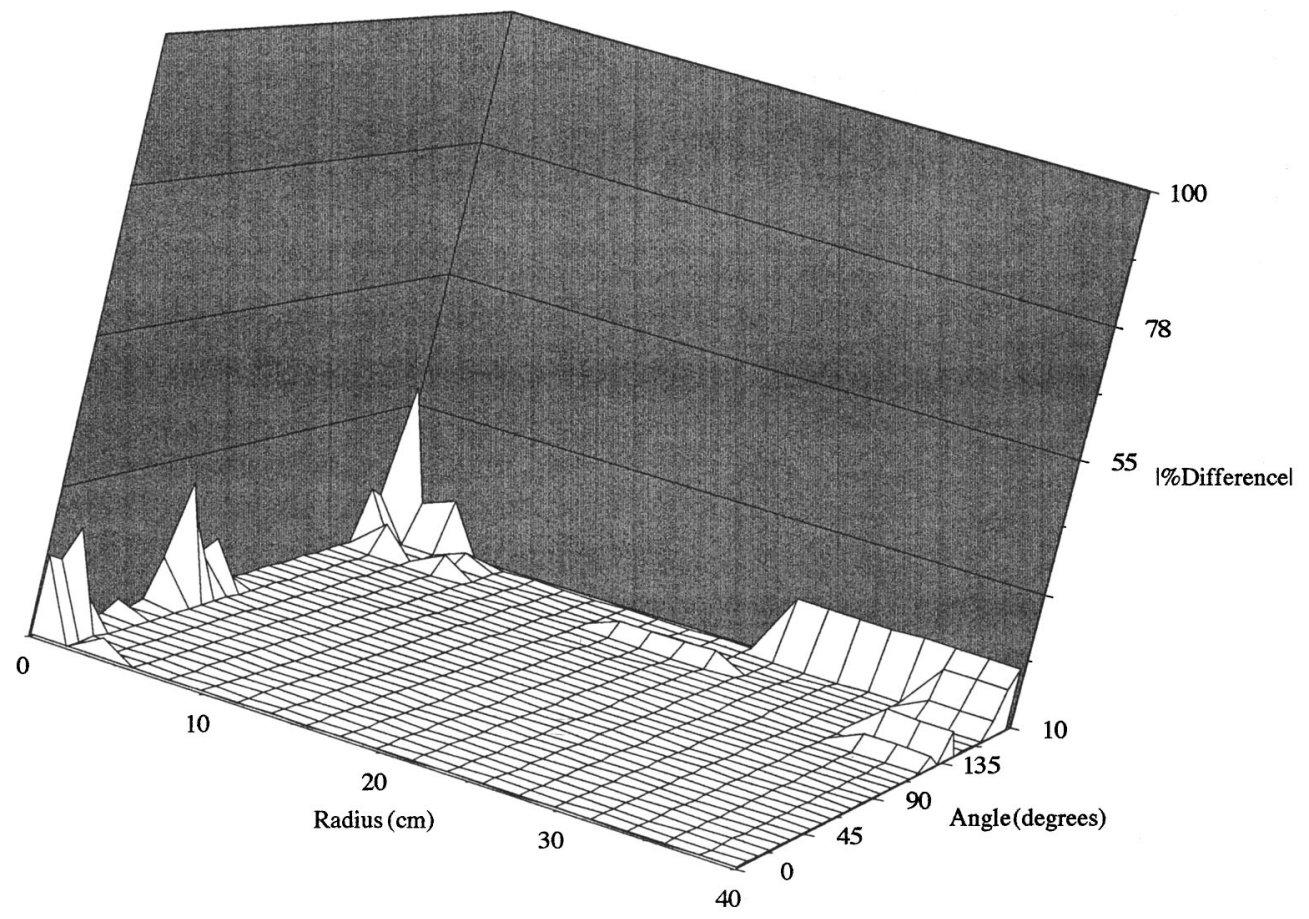

FIG. 3. Comparison of the finite-range analytic model and Monte Carlo results for the $1.25 \mathrm{MeV}$ first scatter dose spread array. The absolute differences between models are plotted as a function of radius and scatter angle.

using the zero-range analytic model is significantly different than the MC scatter kernel near the origin as expected due to the finite range of the secondary electrons. Overall, the differences between the first scatter kernels near the origin for the $5 \mathrm{MeV}$ incident photon energy are larger than those of the $1.25 \mathrm{MeV}$ kernel due to the greater scattered photon energy deposited at the interaction points. Similarly, the differ- ences between the finite-range and $\mathrm{MC}$ data are smaller than for the zero-range model near the origin but comparable outside of this region. The finite-range model is, in general, in agreement with the Monte Carlo results with relatively small differences near the origin. With respect to the $1.25 \mathrm{MeV}$ finite-range comparison, the $5 \mathrm{MeV}$ comparison data set contains more voxels with absolute differences greater than 10 .

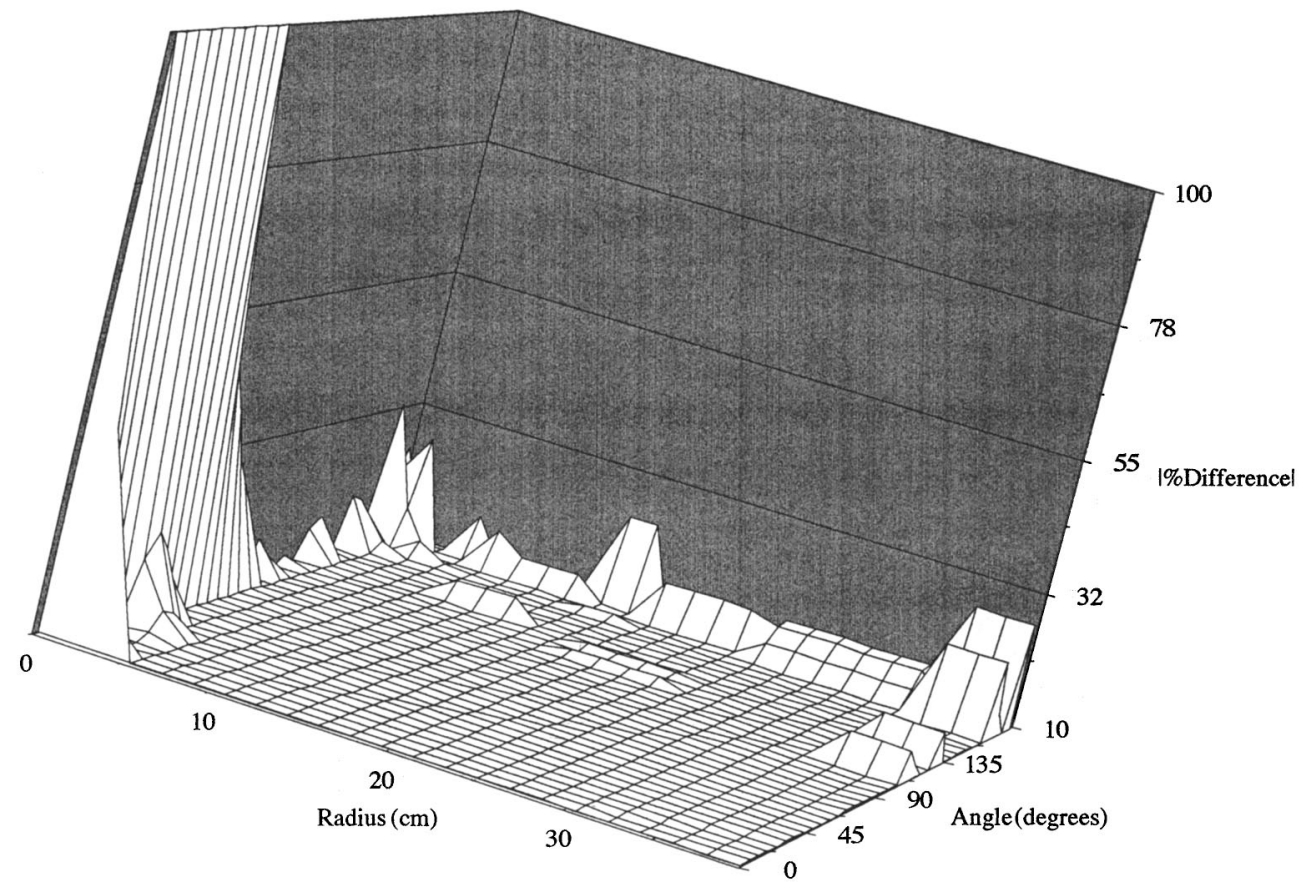

FIG. 4. Differences between the zero-range analytic model and MC data for an incident photon of $5 \mathrm{MeV}$. 


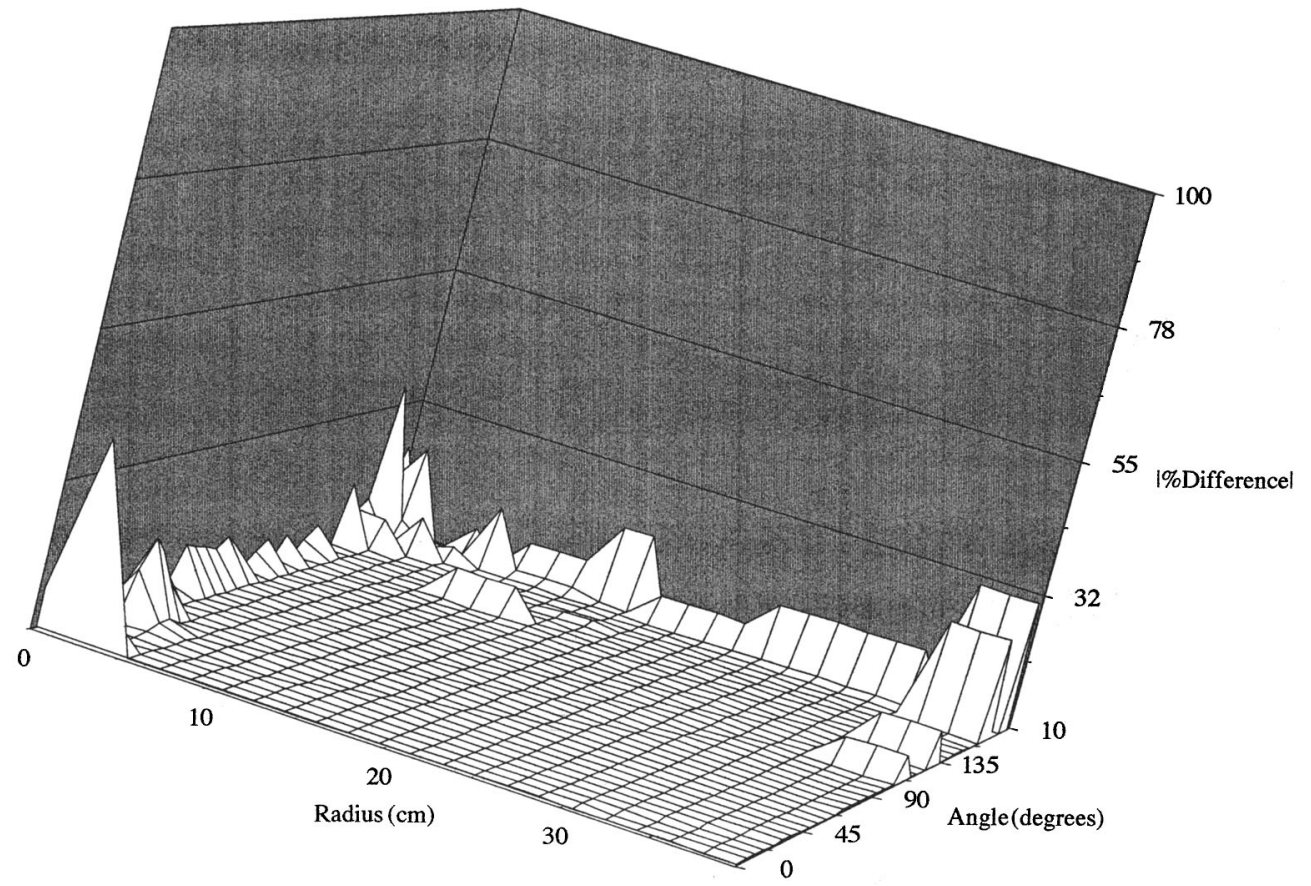

FIG. 5. Differences between the finite-range analytic model and MC data for an incident photon of $5 \mathrm{MeV}$.

Also, there are several voxels in the backscatter region that have actual differences larger than shown in the plots due to the large fluctuations between voxels.

For the $18 \mathrm{MeV}$ incident photon, differences between the point dose spread array calculations using the Monte Carlo and the finite-range model are shown in Fig. 6. The largest differences between the kernels are located near the origin and in the backscatter region where differences range be- tween $10 \%$ and $30 \%$. Again, these results show that as the incident photon energy increases, the differences between the models and Monte Carlo data increase; the magnitude and volume of differences that are larger than $10 \%$ increase with incident photon energy. For all energies considered, the analytic models agree with the Monte Carlo first scatter kernel data to within approximately $10 \%$ for the region bounded by the electron range and backscatter angle.

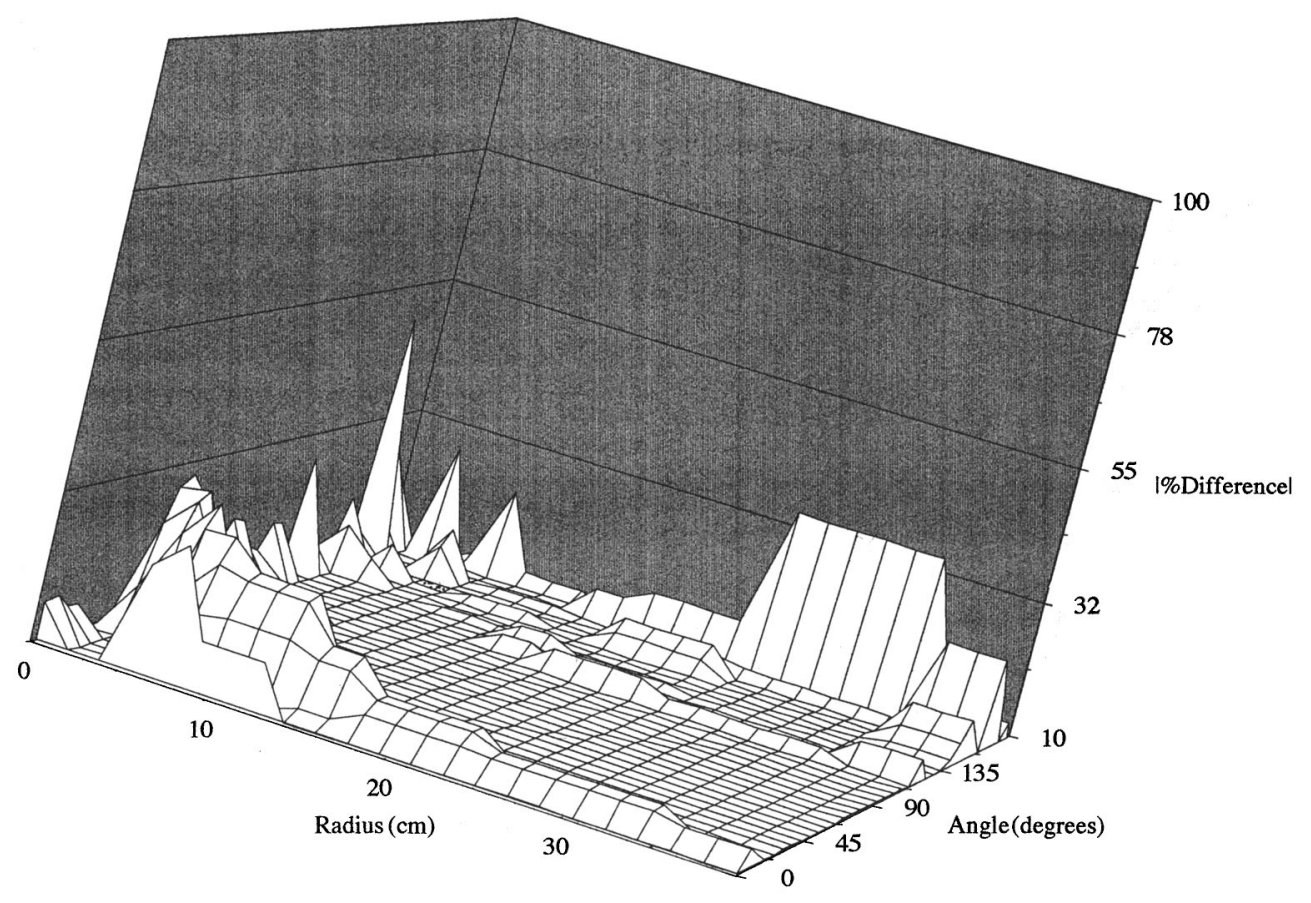

FIG. 6. Differences between the zero-range analytic model and $\mathrm{MC} 18 \mathrm{MeV}$ point dose spread array. 


\section{DISCUSSION}

The analytic and Monte Carlo model calculations agree to within $\sim 10 \%$ for the volume limited by forward scatter photons at radial distances larger than the charged particle range, where the range depends on the scattered photon energy. In the backscatter region, there are voxels that exhibit fairly large discrepancies between the MC and zero-range model results; the differences between the calculations increase in magnitude with increasing Compton photon scatter angle, as seen in the plots for radii larger than $30 \mathrm{~cm}$. The character of these differences appears to contradict the expected behavior of the zero range model as a function of scatter angle where the accuracy should increase as a function of angle. Energy deposited at point should be a valid approximation to electronic equilibrium for conditions where the electron range is small compared to the dimensions of the voxel. As the scattered photon energy decreases with Compton scatter angle, the associated secondary electron range decreases. In addition, the voxel size increases with the angle making the ratio of electron range to voxel size decrease as a function of scatter angle. Therefore, the absolute differences between the zero-range model and Monte Carlo results should also decrease as a function of angle; however, the opposite trend is seen in some of the voxels within the backscatter region.

The zero range model is expected to agree with the Monte Carlo results for conditions that approximate electronic equilibrium and is not expected to be a reliable comparison for regions where nonequilibrium exists. Considering that the shell width is the smallest dimension of the voxel, lateral equilibrium should be present for voxels that consist of shell widths that are approximately equal to or larger than the range of secondary electrons generated by the Compton scattered photons. The zero-range model should agree with the Monte Carlo results for a range of radii and scatter angles where the ratio of electron range to shell width is less than 1 . As an example, this condition should exist for radii greater than $3 \mathrm{~cm}$ for an incident photon of $1.25 \mathrm{MeV}$. As the scatter angle increases, the Compton photon energy decreases and approximate equilibrium conditions should exist at smaller radial distances away from the primary interaction site. At $90^{\circ}$, equilibrium conditions should exist for radii larger than about $0.5 \mathrm{~cm}$. Similarly, for an incident photon of $5 \mathrm{MeV}$, conditions for lateral equilibrium should be present for radii larger than $15 \mathrm{~cm}$ at small scatter angles and for radial distances greater than $2 \mathrm{~cm}$ for scatter angles larger than 50 . In general, the agreement between the Monte Carlo and zero range model results is within $10 \%$ for regions where the ratio of electron range to shell width is less than 1 . There are some exceptions, as mentioned before, where voxels in the backscatter region should be in good agreement but are not.

The strict criteria for lateral equilibrium based upon the ratio of electron range to voxel width establishes a confidence level for comparison. However, the model predictions are not expected to deviate significantly from the Monte Carlo results in regions where there is some degree of nonequilibrium and the criteria is not completely satisfied. An example of partial equilibrium is where the electron range is larger than the shell width but much less than the voxel length. For an incident photon of $5 \mathrm{MeV}$, this condition exists for voxels between 10 and $15 \mathrm{~cm}$ at $0^{\circ}$. The comparison results show that the zero-range model agrees with the Monte Carlo results to within $10 \%$ in this region of partial equilibrium. For radial distances larger than $15 \mathrm{~cm}$, where the voxel width is comparable to the electron range, the agreement is better where the difference between models is not larger than 6\%. Differences are not expected to be substantially larger in regions of partial equilibrium and the comparisons should reflect this behavior.

The region for which the finite-range analytic model is not expected to agree with the Monte Carlo values is near the origin where nonequilibrium exists and electron transport needs to be considered. Within this region, the zero-range model assumes that electrons deposit their total energy at the Compton photon interaction site and can overestimate energy deposition by up to four orders of magnitude. To provide a better estimate within this region, the finite-range model calculates the energy deposition as though electrons deposit energy linearly along a radial path away from the primary interaction site. This is not intended to accurately model the physical interactions in this region, but as a method to estimate kernel values to within a factor of approximately 100 . Electrons generated in the calculation voxel will deposit energy along a path length that is on the order of a voxel width and scatter out of the volume. For this condition, the calculation overestimates the energy contribution by including the radial path length from the Compton electron creation point to the distal end of the voxel. This approximation overestimates the energy deposition by up to a factor of $\sim 10$ due to the ratio of voxel length to width for the calculation geometry considered here. Conversely, the underestimated contribution from electrons generated outside of the calculation voxel is not included. The contribution from the surrounding voxels is complicated; the number of electrons scattered into the calculation voxel depends on many factors including the scattered photon number, electron energy, and electron scatter angles. Based solely upon the number of scattered photons outside of the calculation voxel, the maximum number of electrons generated from first scattered Compton photons will be a factor of 100 larger than that created within the calculation voxel. Using these estimates, the expected maximum deviation from the Monte Carlo data is expected to be less than a factor of 100 for the first voxel outside the primary interaction site. This is seen in the comparisons between the MC and finite-range model results, except for several voxels in the backscatter region.

In general, the analytic first scatter kernel agrees with the Monte Carlo model data where the analytic approximations are shown to be appropriate. There is some unexpected disagreement between the model results in the backscatter region which indicates that there might be some inaccuracies within this specific Monte Carlo data set used for these comparisons - there are voxels exhibiting significant differences between model predictions in the regions where the approximations used in the analytic model should be valid. The observation that these differences are associated with 


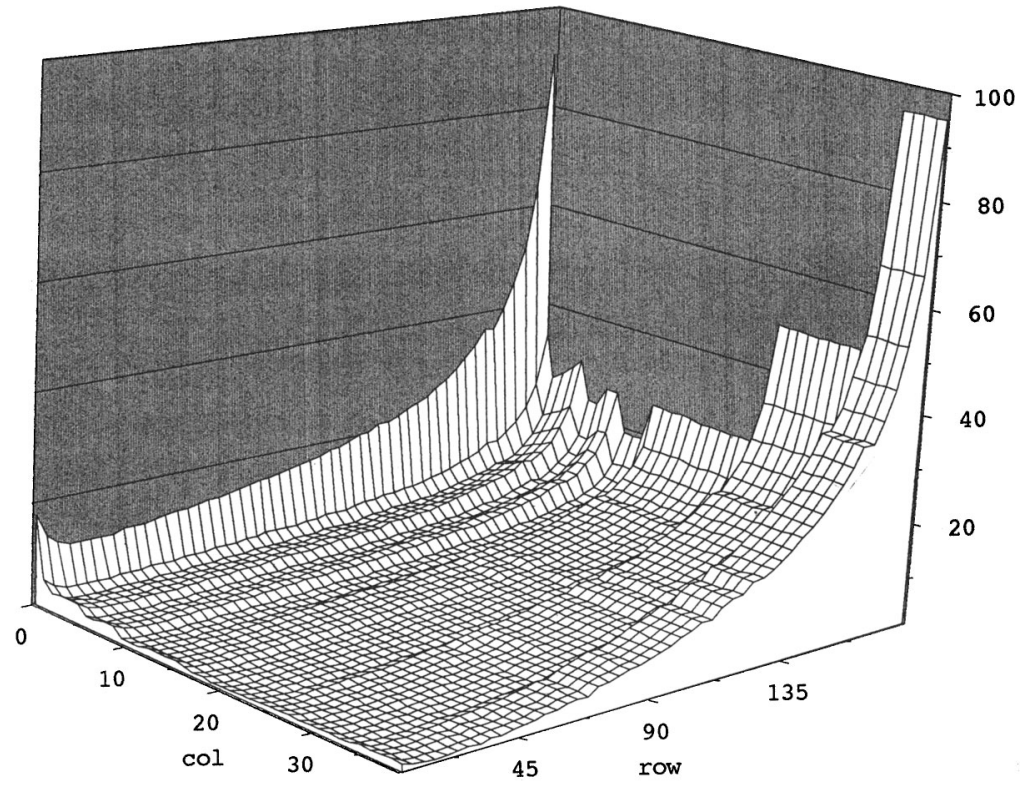

Fig. 7. Plot of relative variation for the first scattered Compton photon-electron creation events from an incident $5 \mathrm{MeV}$ photon.

large fluctuations in the Monte Carlo data implies that the source of error may be related to the random variations of events within a voxel.

To investigate this possibility, the relative variation in electrons created within a voxel by first scattered Compton photons were calculated. As an example of these calculations, Fig. 7 shows the relative variation of Compton photon-electron events for an incident $5 \mathrm{MeV}$ photon. Variations in the backscatter region are large and are comparable to the number of electron creation events in several sections. The largest variations, where the fluctuations are approximately equal or larger than the event number, occur in the area bounded by radii greater than $30 \mathrm{~cm}$ and scatter angles larger than $150^{\circ}$. Near the origin, the fluctuations increase with angle and become comparable to the event number at $180^{\circ}$. As discussed previously, the differences between the Monte Carlo and zero-range models are also largest in these areas. Furthermore, the behavior of the relative variation with respect to radius and angle is similar to that shown in the difference plots, as shown in Fig. 4 for this example. In addition to the observation that large variations exist in the Monte Carlo backscatter data, the correlation between the relative variation and the model differences indicates that the inaccuracies in this Monte Carlo calculation are due to statistical fluctuations. In this particular case, the analytic models are able to identify some of the errors present in the Monte Carlo data and suggest a possible explanation; however, these models are only intended to verify data with limited accuracy.

With regard to the total energy deposition kernel, the kernel resembles the primary point dose spread array near the primary photon interaction site, and beyond the primary electron range the kernel behaves like the first scattered dose spread array. At larger distances, the multiple scattered photon dose array becomes important and the complete kernel resembles this component. The first scattered photon kernel contribution is orders of magnitude smaller than the primary dose spread array near the primary interaction site. From the previous comparisons, it appears that the zero-range model may be sufficient for validating the first scatter dose spread array with respect to the total energy deposition kernel.

\section{CONCLUSION}

We have developed two analytic models for direct comparison of Monte Carlo generated first Compton scattered point dose spread arrays. The analytic models are simple and consider two limiting conditions: energy deposited at a point, and energy deposited linearly outward from the photon interaction point. Due to the approximations considered here, the models have limited regions of accuracy within the dose spread array volume. The zero-range model, where energy is deposited at the scatter photon-electron interaction point, is valid for ranges that extend beyond the primary electron range. Within one voxel of the primary interaction site, the finite-range model estimates the energy deposition to within two orders of magnitude to identify large errors that might be present within the MC data.

Comparisons are made between the analytic models and specific dose spread arrays generated by Mackie et al. ${ }^{5}$ The results show that there is good agreement for the forward scatter volume but exhibit significant differences within the backscatter region where the analytic model assumptions are appropriate. With the specific data used for comparisons in this paper, the analytic model suggests that the Monte Carlo data may be in error due to statistical fluctuations in the first scatter photon-electron creation events. From these examples, the analytic model demonstrates its use for identifying large errors in first scatter dose spread arrays.

\section{ACKNOWLEDGMENT}

This work was supported by Grant No. CA64307 from the National Cancer Institute. 
${ }^{\text {a) }}$ Currently at the Department of Radiology, Baylor College of Medicine, Houston, TX 77030

${ }^{b)}$ Currently at the Division of Radiation Physics, Stanford University School of Medicine, Stanford, CA 94305-5105. Electronic mail: i:boyer@reyes.stanford.edu

${ }^{1}$ T. R. Mackie, J. W. Scrimger, and J. J. Battista, "A convolution method of calculating dose for 15-MV x rays," Med. Phys. 12, 188-196 (1985).

${ }^{2}$ A. Boyer and E. Mok, "A photon dose distribution model employing convolution calculations," Med. Phys. 12, 169-177 (1985).

${ }^{3}$ R. Mohan, C. Chui, and L. Lidofsky, "Differential pencil beam dose computation model for photons,"' Med. Phys. 13, 64-73 (1986).

${ }^{4}$ A. Ahnesjo, P. Andreo, and A. Brahme, "Calculation and application of point spread functions for treatment planning with high energy photon beams," Acta Oncol. 16, 49-56 (1987).

${ }^{5}$ T. R. Mackie, A. F. Bielajew, D. O. Rogers, and J. J. Battista, "Generation of photon energy deposition kernels using the EGS4 Monte Carlo code,"' Phys. Med. Biol. 33, 1-20 (1988).

${ }^{6}$ J. E. McGary and A. L. Boyer, "An interactive, parallel, three dimensional fast Fourier transform calculation using a supercomputer," Med. Phys. 24, 519-521 (1997).
${ }^{7}$ A. Anesjho, "Application of transform algorithms for calculation of absorbed dose in photon beams," 8th International Conference on Computers in Radiotherapy, 17-20 (1984).

${ }^{8} \mathrm{C}$. Chui and R. Mohan, "Extraction of pencil beam kernels by the deconvolution method," Med. Phys. 15, 138-144 (1988).

${ }^{9}$ C. P. Ceberg, B. E. Bjarngard, and T. C. Zhu, "Experimental determination of the dose kernel in high-energy x-ray beams," Med. Phys. 23, 505-511 (1996).

${ }^{10}$ J. E. O'Connor and D. E. Malone, "A cobalt-60 primary dose spread array derived from measurements," Phys. Med. Biol. 34, 1029-1042 (1989).

${ }^{11}$ C. X. Yu, T. R. Mackie, and J. W. Wong, "Photon dose calculation incorporating explicit electron transport," Med. Phys. 22, 1157-1165 (1996).

${ }^{12}$ J. W. Wong and R. M. Henkelman, "A new approach to CT pixel-based dose calculation in heterogeneous media," Med. Phys. 10, 199-208 (1983).

${ }^{13}$ H. E. Johns and J. R. Cunningham, The Physics of Radiology (Charles C. Thomas, Springfield, IL, 1983) 